\title{
Die Schmelzen von Kaliumchlorid mit Silberchlorid und den Kaliumchromaten.
}

\author{
Von \\ S. ŻEMOżUżNY. ${ }^{1}$
}

Mit $\mathbf{3}$ Figuren im Text und 1 Tafel.

Bis in die neueste Zeit hinein wurde zur Erforschung der Salzschmelzen ausschliefslich die Schmelzmethode angewandt, hingegen die Frage nach deren Mikrostruktur seltsamerweise aufser Acht gelassen."

Dies liegt wohl einerseits an der Schwierigkeit gute Schliffe zu gewinnen, andererseits an dem Brauche, diese Schliffe unter dem Mikroskop bei durchfallendem Licht zu untersuchen, wie es bei der Untersuchung der Gesteine geschieht.

Es ist herrorzuheben, dals die Untersuchung der Salzschliffe bei durchfallendem Licht grofse Schwierigkeiten bietet. Sehr dỉnne Strukturelemente des Eutektikums haben, wie aus den beigefugten Mikrophotographien ersichtlich, die Dimensionen von $1 / 200$ bis 1/400 mm:

Um dieselben im durchfallenden Lichte sichtbar zu machen, mufs man Schliffe ron derselben Dickenordnung herstellen; sonst werden diese Flemente ubereinander lagern. Die Herstellung solcher Schliffe bei Salzschmelzen macht aufserordentliche Schwierigkeiten.

Viel einfacher ist es, Schliffe flur reflektiertes Licht herzustellen; dann kommt es auf die Dicke nicht an und die feinen Strukturelemente treten ziemlich deutlich hervor.

1 Ins Dentache tbertragen von J. Pnsskes-Berlin. Erschienen in den Answ. d. Petersb. Polytechn. Institut 6 (1906), 443.

- Nur in der Arbeit von R. LoREirz und W. Rooxaruhe, thber Kgliumbleichloride, $Z$. anorg. Chem. 51 (1906), 71; findet sich neben dem Schmelzdiagramm eine ausfuhrliche krystallographische Untersuchung angegeben. 
In der Abhandlung: „Porphyrartiges Gefige und Eutektikum“ wendete ich und F. Lewinson-Lessing ${ }^{1}$ unser gemeinsames Interesse der Mikrostruktur der Salzschmelzen zu und zeigten, dafs sie sich von der Struktur der Metallegierungen nur unwesentlich unterscheidet. Die Gestze, welche die Kristallisation der geschmolzenen Gemische beherrschen, sind ebenso wie auf die Metallegierungen auch auf Salze und auf Silikate anwendbar; daher muls die Struktur der einen wie der anderen in analogen Fällen die gleichartige sein.

In der vorliegenden Arbeit wird zur Untersuchung der Salzschmelzen das Studium deren Mikrostruktur zugleich mit dem Studium des Schmelzdiagramms angewendet.

Als Ausgangsmaterial dienten reine Salzpräparate von KAHLBAUM. Es wurden $35-50 \mathrm{~g}$ angewandt. Das Gemisch der vorber ausgegluhten Salze: worde in einen Porzellantiegel eingetragen, der sich in einem Graphittiegel befand, wobei der Zwischenraum zwischen deren Wänden mit feinem Sand ausgefullt war. Der Schmelzprozels wurde im Fletscherofen ausgeführt. Nach Beendigung der Schmelzoperation wurde der Tiegel mit einem verschiebbaren Deckel aus feuerbeständigem Ton bedeckt und der Ofen geschlossen.

In das geschmolzene Gemisch wurden die Drähte des Thermoelementes eingetaucht und die Bestimmung der Schmelatemperaturen geschah mit Hilfe des Registrierpyrometers des Herrn Prof. N. KonNAKow. ${ }^{2}$ Vor jeder Versuchsreihe wurde der Apparat graduiert; zu diesem Zwecke wurden auf das lichtempfindliche Papier entsprechende Grundlinien aufgetragen; als solche dienten die Schmelztemperaturen des $\mathrm{Ag}\left(962^{\circ}\right), \mathrm{Sb}\left(631^{\circ}\right), \mathrm{Zn}\left(419^{\circ}\right), \mathrm{Pb}\left(327^{\circ}\right) ; \mathrm{Sn}\left(232^{\circ}\right)$.

\section{Die Schmelzen von $\mathrm{KCl}+\mathrm{K}_{2} \mathrm{CrO}_{4}$.}

Das nentrale Kaliumchromat ändert beim Erbitzen seine Farbe rom gelb in rot, wobei es stark dekrepitiert. Beim Auskrystallisieren aus dem geschmolzenen Zustande scheidet sich das $\mathrm{K}_{2} \mathrm{CrO}_{4}$ in roten Krystallen aus, die nachber die normale gelbe Farbe annehmenen.

Eine derartige Erscheinung wurde schon längst beobachtet und in Zusammenhang mit der Existenz einer zweiten Modifikation gebracht, deren Umwandlungstemperatur nicht ermittelt wurde.

I S. Żemczožny und F. Lewingon-Lessing, Ann. d. Polyt. Inst. 5 (1906), 207.

Ann. d. Polyt. Inst. 1904, 1 ; Journ. russ. phys,-chem. Ges. 1904; Z. anorg. Ohem. 42 (1904), 184. 
Die Bestimmungen mit Hilfe des Registrierpyrometers ergaben, dafs diese Umwandlung in die zweite Modifikation sich bei $679^{\circ}$ vollzieht, wobei die Schmelztemperatur zu $984^{\circ}$ erhalten wurde, was etwas von $L_{E}$ Chatetrme s ${ }^{1}$ Bestimmung abweicht, der $975^{\circ}$ angibt. Der Haltepunlrt auf der der Umwandlung in die zweite Modifikation entsprechenden Abkühlungskurve ist von ziemlich grofser Dauer und der Zeit nach fast um das doppelte kleiner als der Haltepunkt, der der Krystallisation aus der Schmelze entspricht.

Die ziffermälsigen Daten, die sich auf die Bestimmung der Schmelztemperaturen bezieben, sind in Tabelle 1 und Schmelzdiagramm Fig. 1 wiedergegeben. Das Schmelzdiagramm besteht aus 2 Zweigen; die sich im eutektischen Punkte bei $658^{\circ}$ schneiden, der der Zusammensetzung von 31.5 Molekularproz. $\mathrm{K}_{2} \mathrm{CrO}_{4}$ (68.5 Molekularproz. $\mathrm{KCl}$ ) entspricht.

Tabelle 1.

Schmelztemperaturen von $\mathrm{KCl}+\mathrm{K}_{\mathbf{q}} \mathrm{CrO}$.

\begin{tabular}{l|c|c|c}
\hline \hline $\begin{array}{c}\text { Molekular- } \\
\text { prozente }\end{array}$ & $\begin{array}{c}\text { Beginn der } \\
\text { Krystallisation } \\
\text { in }\end{array}$ & $\begin{array}{c}\text { Eutektischer } \\
\text { Haltepunkt } \\
\text { in }\end{array}$ & $\begin{array}{c}\text { Modifikationen } \\
\text { in }\end{array}$ \\
\hline \hline $\mathrm{KCl}$ & 790 & & \\
$1.78 \mathrm{~K}_{\mathbf{9}} \mathrm{CrO}_{4}$ & 781.5 & 656 & \\
4.19 & 770.5 & 656 & \\
10.9 & 745 & 657 & \\
16.95 & 720 & 658 & \\
28.52 & 694 & 658 & \\
80.00 & 664 & 658 & 676 \\
38.50 & 682 & 658 & 676 \\
46.93 & 718 & 658 & 676 \\
53.52 & 768 & 658 & 676 \\
60.00 & 804 & 658 & 678 \\
70.08 & 864 & 658 & 676 \\
75.50 & 886 & 658 & 676 \\
78.00 & 906 & 658 & 676 \\
80.06 & 916 & 658 & 676 \\
89.80 & 958 & 658 & 679 \\
91.90 & 960 & 657 & \\
$\mathrm{~K}_{\mathbf{8}} \mathrm{CrO}$ & 984 & & \\
& & & \\
& & & \\
\hline
\end{tabular}

Die Abkublungskurven auf dem Zweige $A B$ haben 2 Haltepunkte: der erste bei veränderlicher Temperatur in Abhängigkeit

' Le Chatgusa, Bull. Soo, Ohim. 47 (1887), 300. 
von der Zusammensetzung entspricht der Krystallisation des Chlorkaliums aus dem geschmolzenen Gemische, der 2weite - der eutektische - bei konstanter Temperatur $658^{\circ}$, ist durch das gleichzeitige Auskrystallisieren der beiden Komponenten des Systems charakterisiert.

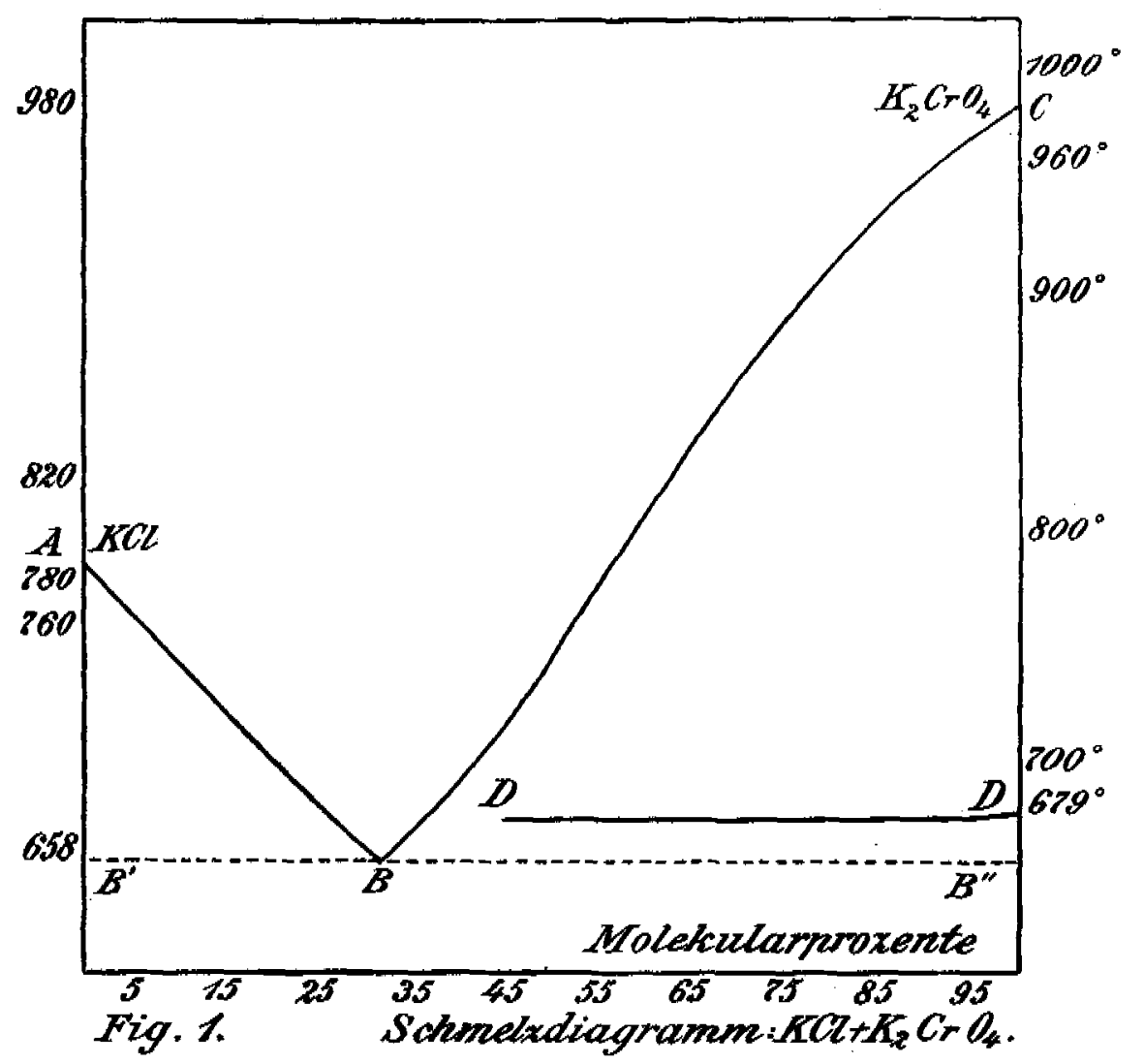

Der eutektische Haltepunkt tritt schon bei dem Gehalt von 1.78 Molekularproz. $\mathrm{K}_{2} \mathrm{CrO}_{4}$ auf und ist von ziemlich merklicher Länge, woraus man schliefsen mufs, dals von der Seite des Chlorkaliums sich keine feste Lösungen mit $\mathrm{K}_{2} \mathrm{CrO}_{4}$ bilden; derselbe erreicht sein Maximum im Punkte $B$ bei 31.5 Molekularproz. und fängt weiterhin an abzunehmen.

Der $\mathrm{Zweig} A B$ ist durch die Ausscheidnngen der Kalium. chromatkrystalle charakterisiert, deren Schmelztemperatur mit steigen. dem KCl-Gehalt in der geschmolzenen Masse erniedrigt wird. Die Abkuhlungskurven haben 3 Haltepunkte: der erste entspricht der 
Krystallisation des $\mathrm{K}_{9} \mathrm{CrO}_{4}$, der zweite bei der Temperatur $676^{\circ}$ ist durch die Umwendiung des $\mathrm{K}_{2} \mathrm{CrO}_{4}$ in die zweite Modifikation bedingt. Diese Umwandlung erfolgt für reines $\mathrm{K}_{2} \mathrm{CrO}_{4}$ bei $679^{\circ}$, für seine Gemiriche mit $\mathrm{KCl}$ findet sie schon bei $676^{\circ}$ statt, infolge der Bildung fäster Lösungen von geringer Konzentration.

Unterhalb des der Umwandlung in die 2. Modifikation entsprechenden Haltepunktes lälst sich auf den Abklihlungskurven ein dritter Haltepunkt $B$, ein eutektischer, bei $658^{\circ}$ beobachten. Bei 10.2 Molekularproz. $\mathrm{KCl}$ ist der eutektisch Haltepunkt von geringer Länge.

Wenn man nach Tamman ${ }^{1}$ auf die Abszissenachse die prozentische Zusammensetzung, auf die Ordinatenachse die Länge der eutektischen Haltepunkte anftrăgt, so ergibt diese Konstruktion für die Länge des entektischen Haltepunktes bei 4 Molekularproz. $\mathrm{KCl}$ den Wert Null. Dies Verhalten weist auf das Vorhandensein fester Lobsungen mit der Grenzkonzentration von etwa 4 Molekularproz. $\mathrm{KCl}$ von der Seite des $\mathrm{K}_{8} \mathrm{CrO}_{4}$ bin. Im Zusammenhang mit der Existenz fester Lősungen steht die Erniedrigung der Umwandlungstemperatur des Kaliumchromates von $679^{\circ}$ auf $676^{\circ}$.

Die Mikrostruktur der Schmelzen steht im vollen Einklang mit den Ergebnissen des Schmelzdiagramms.

Auf dem dex Kristallisation des Chlorkaliums entsprechenden Zweige $A B$ sind KCl-Krystalle im eutektischen Gemische sichtbar, auf dem $\mathrm{Zweige} B C$ scheiden sich $\mathrm{K}_{2} \mathrm{CrO}_{4}$-Krystalle aus, umgeben von der eutektischen Masse. Schliff Nr. 1 (Tafel XVII) entspricht dem Zwoige $A B$. Nach leichter Behandlung dieses Schliffes mit Spiritus treten auf dem Untergrunde des Eutektikums dunkle KCl-Krystalle auf; in Wirklichkeit sind dies jedoch nur Hohlräume, die an Stelle der mit Spiritus herausgelösten Krystalle eutstanden sind. Das Kaliumchromat wird ron Spiritus nicht ausgelaugt, und daher sind auf Schliff Nr. 2 weifse $\mathrm{K}_{2} \mathrm{CrO}_{4}-\mathrm{Krystalle}$ in der eutektischen Masse zu sehen. Der Schliff entspricht dem Zweige $C B$.

\section{Sohmelzen von $\mathrm{KCl}+\mathrm{K}_{2} \mathrm{Cr}_{2} \mathrm{O}_{7}$.}

Kaliumbichromat scheidet sich aus dem geschmolzenen Zustande in dunkelroten Krystallen aus, die bei weiterer Abkühlung etwas heller werden, wobei sie in Pulver unter bedeutender Volumenausdebnung zerfallen.

Z. anorg. Ohem. 45, 24. 
Auf diese Erscheinung wurde zuerst Mirsoneruion ${ }^{1}$ aufmerksam. Aus Erscheinungen dieser Art wurde auf die Existenz einer zweiten Modifikation bei einer Temperatur von etwa $240^{\circ}$ geschlossen. Bei dieser Temperatur erfährt das Salz eine so erhebliche Volumenzunabme, dals das Reagensglas, in dem das Salz schmilzt, zerspringt.

Die Versuche, mit Hilfe des Thermometers die bei diesem Vorgange entwickelte Wärme zu bestimmen, führten zu keinem positiven Resultaten, es wurden keine Haltepunkte in dem Verlaufe der Temperaturänderung beobachtet. Aus diesem Grunde ist die Umwandlung, die das Kaliumbichromat beim Übergang in die zweite Modifikation erfăhrt, zur Kategorie derjenigen Umwandlungen gerechnet worden, die trotz einer bedeutenden Volumzunahme ohne merkliche Energieanderungen vor sich gehen. ${ }^{3}$

Tabelle 2.

Schmelztemperaturen der Schmelzen $\mathrm{KCl}+\mathbf{K}_{\mathbf{9}} \mathrm{CN}_{9} \mathrm{O}_{7}$.

\begin{tabular}{|c|c|c|c|}
\hline $\begin{array}{l}\text { Molekular- } \\
\text { prozente }\end{array}$ & $\begin{array}{c}\text { Beginn der } \\
\text { Krystallisation } \\
\text { in }\end{array}$ & $\begin{array}{c}\text { Eutektischer } \\
\text { Haltepunkt } \\
\text { in }\end{array}$ & $\begin{array}{c}\text { Modifikationen } \\
\text { in }\end{array}$ \\
\hline $\mathrm{K}_{2} \mathrm{Cr}_{2} \mathrm{O}_{7}$ & 395 & & 236 \\
\hline $4.32 \mathrm{KCl}$ & 985 & & 235 \\
\hline 9.31 & 378 & & 234 \\
\hline 15.29 & 371 & & \\
\hline 20.00 & 370 & & \\
\hline 25.00 & 368 & & \\
\hline 80.00 & 381 & 366 & \\
\hline 30.95 & 383 & 366 & \\
\hline 35.00 & 410 & 366 & \\
\hline 41.55 & 458 & 366 & \\
\hline 50.00 & 523 & 366 & \\
\hline 60.00 & 582 & 366 & \\
\hline 70.00 & 642 & 366 & \\
\hline 78.70 & 685 & 366 & \\
\hline 86.42 & 722 & 366 & \\
\hline 93.47 & 757.5 & 365 & \\
\hline 97.45 & 776.5 & 365 & \\
\hline $\mathrm{KCl}$ & 790 & & \\
\hline
\end{tabular}

Das Studium der Abkühlungskurve der $\mathrm{K}_{2} \mathrm{Cr}_{2} \mathrm{O}_{7}$-Schmelze mit Hilfe des Registrierapparates zeigte die Irrtümlichkeit dieser Schlufsfolgerung. Die Abkühlungskurve des Kaljumbichromats hat einen

1 Pogg. Ann. 28, 120.

2 Tammann, Krystallisieren und Schmelzen (1903), S. 40. 
ziemlich deutlich ausgesprochenen Haltepunkt unterhalb der Schmelztemperatur bei $236^{\circ}$.

Mifst man auf dem Diagramm die Längen der Haltepunkte, die dem Schmelzen und dem Übergang in die zweite Modifikation

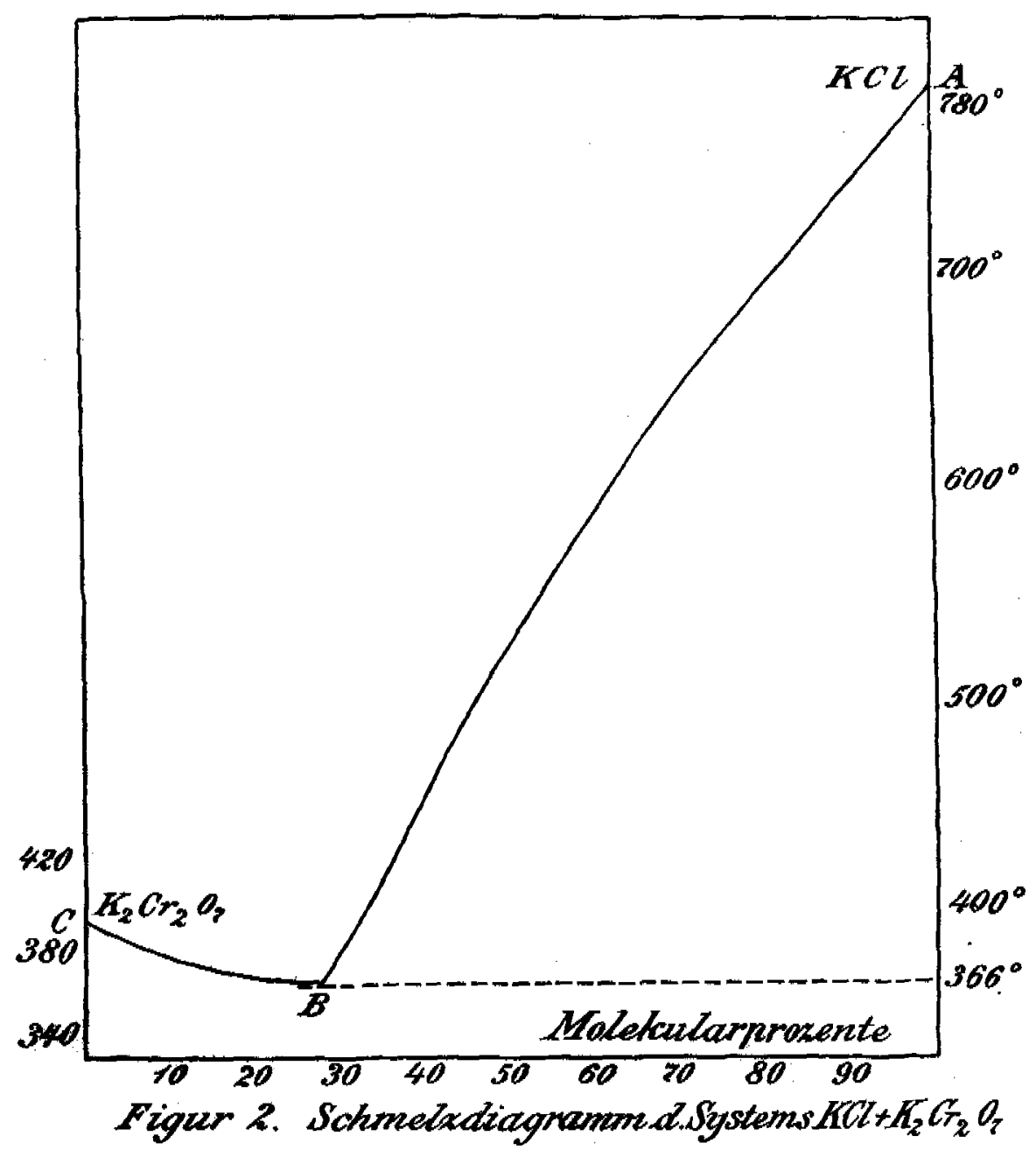

entsprechen, so ergibt sich ibr Verbaltuis zu 20-25 und demgemäfs mufs auch das Verhältnis zwischen den bei diesen Prozessen entwickelten Wärmemengen annähernd dasselbe sein.

Um diese Umwandlung in die zweite Modifikation auf thermischem Wege zu ermitteln, mufs das Thermometer in das geZ. anorg. Chem. Bd. 57, 
schmolzene Salz ohne Hülle eingetaucht werden, anderen Falles bekommt die Haltelinie die Form einer gestreckten Welle; aufserdem mufs die Abkühlungsgeschwindigkeit nicht zu gering sein.

Das Schmeladiagramm besteht aus $\mathrm{zwei} \mathrm{Z}$ weigen $A B$ und $B C$, die sich im eutektischen Punkte $B$ bei $366^{\circ}$ und bei einem Gebalt der Schmelze von 27.5 Molekularprozent $\mathrm{KCl}$ (72.5 Molekularprozent $\mathrm{K}_{8} \mathrm{Cr}_{2} \mathrm{O}_{7}$ ) schneiden. Auf den Abkühlungskurven des Zweiges $A B$, der der Krystallisation des Chlorkaliums entspricht, sind zwei Haltepunkte merkbar; der erste entspricht der Ausscheidung der KCl-Krystalle, der zweite ist der eutektische bei $366^{\circ}$.

Auf dem Zweige $C B$ haben die Abkühlungskurven nur einen Haltepunkt, was auf die Existenz fester Lösungen von Chlorkalium in Kaliumbichromat hinweist. Die Grenzkonzentration dieser festen Lösungen liegt bei ca. 25 Molekularprozent $\mathrm{KCl}$, was den eutektischen Punkt $(27.5 \% \mathrm{KCl})$ fast erreicht.

Die Abkühlungskurve des reinen Kaliumbichromats hat zwei Haltepunkte; der erste entspricht der Krystallisation des Salzes aus der Schmelze bei $395^{\circ}$, der zweite bei $236^{\circ}$ der Umwandlung in die zweite Modifikation.

Auf den dem Zweige $C B$ entsprechenden Schliffen (von der Seite $\mathrm{K}_{2} \mathrm{Cr}_{2} \mathrm{O}_{7}$ ) ist die homogene Struktur der festen Lösung sichtbar.

In den dem $\mathrm{Z}_{\text {weige }} A B$, auf dem die Ausscheidung des Chlorkaliums vor sich geht, entsprechenden Schliffen, sind KCl-Dendrite auf dem Untergrunde des Eutektikums sichtbar, das ùbrigens keine charakteristisohe Struktur besitzt, da feste Lösungen mit einer Grenzkonzentration von 25 Molekularprozent $\mathrm{KCl}$ vorhanden sind, während das Eutektikum die Zusammensetzung von 27.5 Molekularprozent $\mathrm{KCl}$ besitzt.

\section{Schmelzen von $\mathbf{K C l}+\triangle \mathrm{AgCl}$.}

Das Schmelzdiagramm dieses Systemes besteht aus zwei Zweigen $A B$ und $B C$, die sich im eutektischen Punkte $B$ bei $960^{\circ}$ schneiden, der einer Zusammensetzung von 30 Molekularprozent $\mathrm{KCl}+70$ Molekularprozent $\mathrm{AgCl}$ entspricht.

Die ziffernmälsigen Daten, auf Grund deren das Diagramm (Fig. 3) konstruiert ist, sind in Tabelle 3 angefuhrt. Die Schmelztemperatur des Silberchlorids ergab sich $2 \mathbf{4 5}^{\circ}$, was mit den $\mathrm{Be}$. stimmungen von CARNELLEX ${ }^{1}$ übereinstimmt.

\footnotetext{
1 Caraellex, Joum. Ohem. Soc. 39 (1878), 273.
} 
Tabelle 3.

Schmelztemperaturen des Systems $\mathrm{KCl}+\mathrm{AgCl}$.

\begin{tabular}{|c|c|c|}
\hline Molekularprozente & $\begin{array}{c}\text { Beginn der Krystalli- } \\
\text { eation in }\end{array}$ & $\begin{array}{c}\text { Eutektischer Halte- } \\
\text { punkt in }\end{array}$ \\
\hline $\mathrm{AgCl}$ & 451 & \\
\hline $2.57 \mathrm{KCl}$ & 439 & 806 \\
\hline 6.68 & 426 & 806 \\
\hline 11.38 & 405 & 306 \\
\hline 15.94 & 384 & 306 \\
\hline 21.75 & 353 & 806 \\
\hline 25.70 & 329 & 806 \\
\hline 26.2 & $\mathbf{3 3 0}$ & 306 \\
\hline 32.8 & 319 & 806 \\
\hline $\mathbf{8 8 . 3}$ & 383 & 306 \\
\hline 43.86 & 444 & 306 \\
\hline 45.00 & 457 & 306 \\
\hline 47.51 & 474 & 306 \\
\hline 50.12 & 494 & 306 \\
\hline 52.49 & 512 & 306 \\
\hline 54.88 & 585 & 306 \\
\hline 60.00 & 562 & 306 \\
\hline 64.73 & 596 & 306 \\
\hline 72.61 & 638 & 306 \\
\hline 77.81 & 667 & 806 \\
\hline 88.30 & 700 & 306 \\
\hline 88.97 & 730 & 306 \\
\hline 94.52 & 760.5 & 305 \\
\hline $\mathbf{K C l}$ & 790 & \\
\hline
\end{tabular}

Die Abkuhlungskurven der auf dem Zweige $A B$ liegenden Schmelzen haben je zwei Haltepunkte: Der erste entspricht der Krystallisation des Chlorsilbers aus Schmelzen von verschiedener Konzentration, der zweite, der eutektische Haltepunkt bei konstanter Temperatur $306^{\circ}$, ist durch gleichzeitige Krystallisation beider Komponenten des Systems charakterisiert.

Der Zweig $C B$ ist durch Ausscheidung der KC-Krystalle aus der geschmolzenen Masse charakterisiert. Die Abkihlungskurven der auf diesem Zweige liegenden Schmelzen haben je zwei Haltepunkte: der erste entspricht der Ausscheidung des Chlorkaliums, der zweite ist der entektische bei $306^{\circ}$.

Trägt man nach TammanN ${ }^{1}$ auf die Abszissenachse die pro-

1 Tammane, Z. anorg. Chem. 45, 24. 
zentuale Zusammensetzung und auf die Ordinateñ die Länge (Dauer) der eutektischen Haltepunkte für Schmelzen von verschiedener $\mathrm{Zu}$. sammensetzung ein, so wird das Maximum der Zeitdauer dem

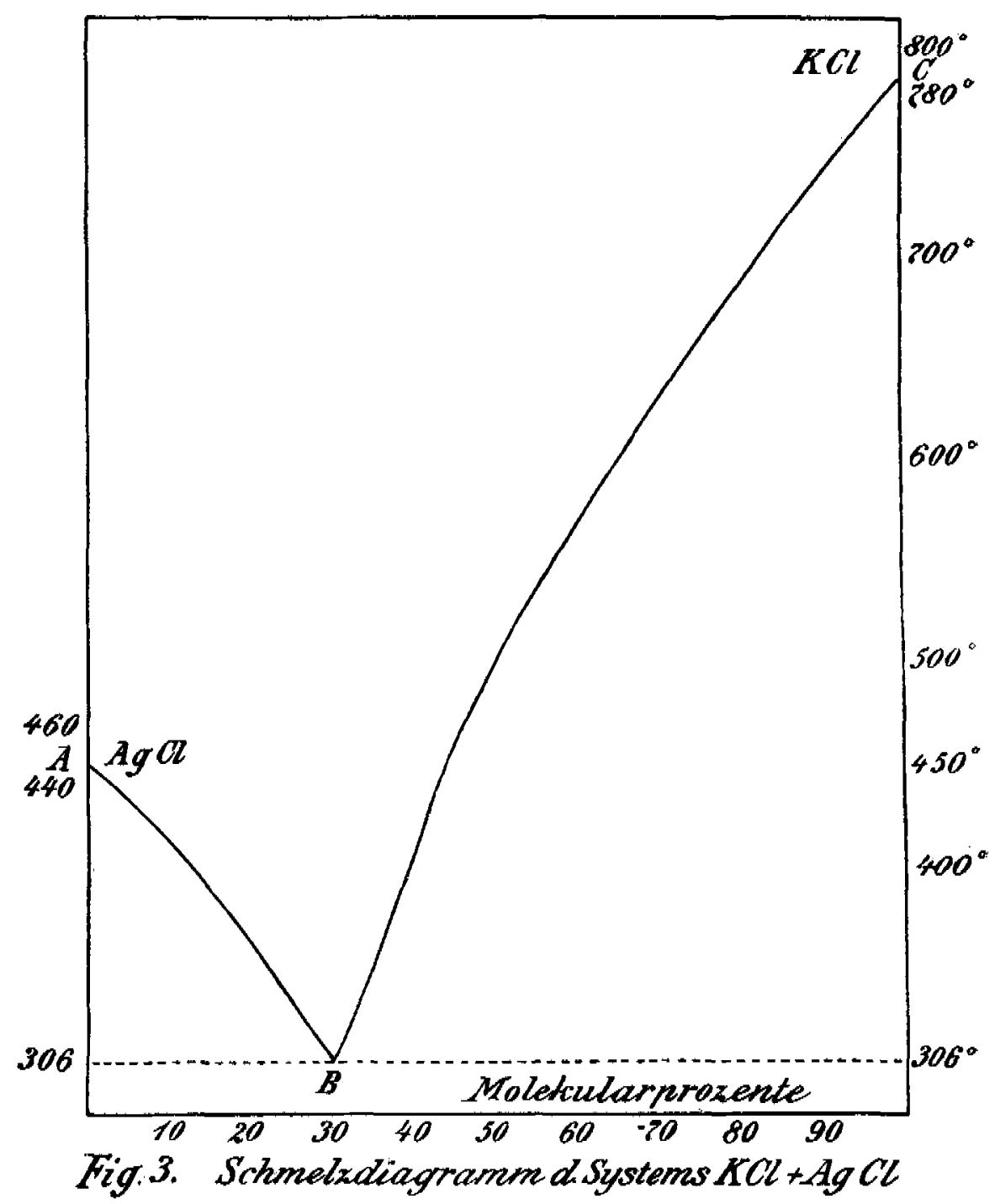

Punkte B (30 Molekularprozent $\mathrm{KCl}$ ) entsprechen; von beiden Seiten des Maximums nimmt die Dauer des eutektischen Haltens ab und wird fast bei den dem reinen $\mathrm{KCl}$ und $\mathrm{AgCl}$ entsprechenden Ordinaten gleich Null. 
Diese Beobachtung deutet an, dafs im vorliegenden System entweder sich keine feste Lösungen oder nur solche von geringer Konzentration bilden.

Die Mikrostruktur der Schmelzen stimmt mit den Resultaten des Diagrammes tiberein. In den dem Zweige $A B$ entsprechenden Schliffen lassen sich Krystalldendriten des Chlorsilbers in dem eutektischen Gemische beobachten; dagegen bei Erstarrung der auf $Z$ weige $B C$ liegenden Schmelzen krystallisiert in der eutektischen Masse Chlorkalinm aus, was sich durch Auftreten von dunklen Chlorkaliumdendriten kennzeichnet.

Schliff Nr. 4 entspricht einer 35 Molekularprozent $\mathrm{KCl}$ enthaltenden Schmelze. Nach Behandlung desselben mit Spiritus werden auf dem Untergrunde des gut ausgebildeten Eutektikums KCl-Dendriten sichtbar.

St. Petorsburg, Polytechn. Institut. Laboratorium fiir allgemeine Chemie.

Bei der Redaktion eingegangen am 11. Dezember 1907. 